\title{
Perspectivas da robótica como recurso pedagógico aplicada a educação 4.0: Uma análise bibliométrica sobre robótica educacional
}

\author{
Perspectives of robotics as a pedagogical resource applied to education 4.0: A bibliometric analysis \\ on educational robotics
}

Perspectivas de la robótica como recurso pedagógico aplicado a la educación 4.0: Un análisis

bibliométrico sobre robótica educativa

Recebido: 14/03/2021 | Revisado: 20/03/2021 | Aceito: 22/03/2021 | Publicado: 31/03/2021

Tamires Fernanda Barbosa Nunes

ORCID: https://orcid.org/0000-0001-5248-1326

Universidade Federal de Santa Maria, Brasil E-mail: tamiresfbnunes@gmail.com

Carol Correia Viana

ORCID: https://orcid.org/0000-0001-9947-5070 Instituto Federal de Educação, Ciência e Tecnologia de Minas Gerais, Brasil E-mail: carolcorreiaviana@gmail.com

Luiz Augusto Ferreira de Campos Viana

ORCID: https://orcid.org/0000-0001-5475-8077 Instituto Federal de Educação, Ciência e Tecnologia de Minas Gerais, Brasil E-mail: luiz.viana@ifmg.edu.br

\begin{abstract}
Resumo
A Quarta Revolução Industrial estendeu a transformação digital para o ambiente educacional, ao reconfigurar o mercado de trabalho e exigir novas habilidades e competências dos profissionais. Desse modo, a Educação 4.0 tem como proposta a inserção de novas tecnologias no ambiente educacional para que o processo de ensino-aprendizagem transcenda a educação tradicional ao impulsionar o ensino inovador e o aprender fazendo, abrindo espaço para uma aprendizagem baseada na experimentação, desenvolvimento de projetos e vivências. Nesse cenário, a robótica educacional se apresenta como um recurso pedagógico capaz de auxiliar a transição tecnológica-digital da educação. O objetivo dessa pesquisa é analisar a robótica educacional como recurso pedagógico capaz de atribuir ao ensino e aprendizagem recursos atuais rumo a Educação 4.0. Para isso, uma análise bibliométrica de coocorrência de palavras foi desenvolvida, visando realizar o levantamento dos principais termos referentes a robótica educacional presentes na literatura e compreender a expansão da robótica como recurso pedagógico ao longo dos anos. Os resultados demonstraram que a robótica como recurso pedagógico encontra-se em plena expansão promovendo a aprendizagem por experimentação, estando associada ao pensamento computacional, as teorias de aprendizagem construtivistas e construcionistas e promovendo o ensino baseado na resolução de problemas. Caracterizando-se como recurso pedagógico atual capaz de proporcionar um ambiente de aprendizagem multidisciplinar favorecendo a integração digital na vida cotidiana dos alunos e a incorporação de habilidades e atitudes capazes de prepará-los para o exercício pleno da cidadania em uma sociedade em constante evolução.
\end{abstract}

Palavras-chave: Robótica educacional; Educação 4.0; Educação digital; Educação tecnológica.

\begin{abstract}
The Fourth Industrial Revolution extended digital transformation to the educational environment by reconfiguring the labor market and requiring new skills and skills from professionals. Thus, Education 4.0 proposes the insertion of new technologies in the educational environment so that the teaching-learning process transcends traditional education by boosting innovative teaching and learning by doing, opening space for learning based on experimentation, project development and experiences. In this scenario, educational robotics presents itself as a pedagogical resource capable of assisting the technological-digital transition of education. The objective of this research is to analyze educational robotics as a pedagogical resource able to attribute to teaching and learning current resources towards Education 4.0. For this, a bibliometric analysis of word co-occurrence was developed, aiming to survey the main terms related to educational robotics present in the literature and understand the expansion of robotics as a pedagogical resource over the years. The results showed that robotics as a pedagogical resource is in full expansion promoting learning by experimentation, being associated with computational thinking, constructivist and constructionist learning theories and promoting teaching based on problem solving. Characterized as a current pedagogical resource capable of providing a multidisciplinary learning environment favoring digital integration in the daily life of students and the
\end{abstract}


incorporation of skills and attitudes able to prepare them for the full exercise of citizenship in a constantly evolving society.

Keywords: Educational robotics; Education 4.0; Digital education; Technological education.

\begin{abstract}
Resumen
La Cuarta Revolución Industrial extendió la transformación digital al entorno educativo, reconfigurando el mercado de trabajo y exigiendo nuevas habilidades y competencias de los profesionales. De ese modo, la Educación 4.0 tiene como propuesta la inserción de nuevas tecnologías en el ambiente educacional para que el proceso de enseñanzaaprendizaje trascienda la educación tradicional al impulsar la enseñanza innovadora y el aprendizaje haciendo, abriendo espacio para un aprendizaje basado en la experimentación, desarrollo de proyectos y vivencias. En este escenario, la robótica educativa se presenta como un recurso pedagógico capaz de auxiliar la transición tecnológicadigital de la educación. El objetivo de esta investigación es analizar la robótica educativa como recurso pedagógico capaz de asignar a la enseñanza y aprendizaje recursos actuales rumbo a Educación 4.0. Para ello, se desarrolló un análisis bibliométrico de coincidencia de palabras, con el objetivo de realizar el levantamiento de los principales términos referentes a la robótica educativa presentes en la literatura y comprender la expansión de la robótica como recurso pedagógico a lo largo de los años. Los resultados demostraron que la robótica como recurso pedagógico se encuentra en plena expansión promoviendo el aprendizaje por experimentación, estando asociada al pensamiento computacional, las teorías del aprendizaje constructivista y constructivo y la promoción de la enseñanza basada en la resolución de problemas. Caracterizándose como recurso pedagógico actual capaz de proporcionar un ambiente de aprendizaje multidisciplinar favoreciendo la integración digital en la vida cotidiana de los alumnos y la incorporación de habilidades y actitudes capaces de prepararpara el ejercicio pleno de la ciudadanía en una sociedad en constante evolución.
\end{abstract}

Palabras clave: Robótica educativa; Educación 4.0; Educación digital; Educación tecnológica.

\title{
1. Introdução
}

A educação é um conceito genérico utilizado para caracterizar as práticas e atividades pelas quais grupos de indivíduos promovem o desenvolvimento intelectual e social de seus membros, permitindo o pleno funcionamento dos mecanismos essenciais da evolução da espécie humana (Coll, 1999). As práticas educacionais englobam os processos de ensino e aprendizagem que representam um sistema complexo de interações comportamentais entre educadores e educandos (Botomé \& Kubo, 2001).

O processo educativo permite que o indivíduo, além de conhecer os elementos que o cercam, possa intervir sobre eles ampliando sua liberdade e comunicação com o ambiente (Saviani, 2000). Desta forma, torna-se indispensável que o processo de aprendizagem se dê de forma contínua, acompanhando o desenvolvimento dos indivíduos e da sociedade.

O desenvolvimento da humanidade possui caráter contínuo. A inovação e os avanços tecnológicos modificam, a cada dia, a vida e o modo como a sociedade interage entre si nas mais variadas esferas. Atualmente, a Quarta Revolução Industrial ou Indústria 4.0 têm transformado o modo como o indivíduo interage com o meio, ao promover soluções digitais inteligentes capazes de associar o mundo físico, digital e biológico por meio de um conjunto de tecnologias (Frank et. al., 2019). A revolução não se limita ao contexto industrial ou da manufatura, se expandindo ao setor de serviços, saúde, educação, entre outros.

A extensão da Indústria 4.0 para o ambiente educacional, conhecida como Educação 4.0, apresenta novos paradigmas para o ambiente escolar ao trazer tecnologias e recursos digitais para o processo de ensino e aprendizagem, acarretando novos desafios e oportunidades para o sistema educacional. A inserção de novas tecnologias no ambiente educacional requer educadores habilitados com práticas pedagógicas interativas, metodologias ativas, domínio de recursos digitais e uma gestão escolar capaz de contribuir para um ambiente colaborativo de aprendizagem (Führ, 2019).

O processo educacional num contexto digital conta com uma ampla variedade de recursos digitais capazes de contribuir para o processo de aprendizagem. A robótica educacional é um exemplo de recurso pedagógico capaz de contribuir para a construção de um modelo educativo inovador e eficaz (Bezerra et. al., 2015).

A Robótica é a ciência ou estudo da tecnologia associado com projeto, fabricação, teoria e aplicação dos robôs. Para 
D'Abreu (2014), a robótica tem tomado uma nova forma, deixando de ser eminentemente para a produção de robôs para se constituir um novo mediador no processo de ensino-aprendizagem. A robótica como recurso pedagógico tem se mostrado eficiente ao possibilitar que o aluno construa o conhecimento por meio da experimentação (Fracasso et. al., 2018).

Diante do exposto, este artigo tem como objetivo analisar a robótica educacional como recurso pedagógico capaz de proporcionar um ambiente de ensino e aprendizagem atual, eficiente e direcionado a evolução do sistema educacional ruma à educação 4.0. Para tal, realizou-se uma análise bibliométrica visando observar a evolução das produções científicas sobre robótica educacional e, por meio da análise de coocorrência, levantar os principais termos referentes a robótica educacional visando associá-los as suas potenciais aplicabilidades no contexto de educação digital.

\section{Revisão Bibliográfica}

Nesta sessão é apresentado um embasamento teórico sobre as teorias da aprendizagem, robótica educacional e educação 4.0, que nortearam a construção do estudo proposto.

\subsection{Teorias da aprendizagem}

Teorias representam tentativas humanas de tornar o conhecimento, de várias áreas, sistemático. Uma teoria de aprendizagem consiste em "uma construção humana para interpretar sistematicamente a área de conhecimento que chamamos de aprendizagem" (Moreira, 1999, p. 12), buscando explicar seu significado. Ao longo dos anos, diversas abordagens foram desenvolvidas visando a otimização do processo ensino-aprendizagem, em virtude das várias transformações que o ambiente educacional sofreu.

O currículo em espiral proposto por Jerome Bruner, no qual um conteúdo é apresentado ao aluno e reforçado continuamente até ser compreendido, influenciou a construção de muitos avanços tecnológicos ao oportunizar que um tópico seja visto em diferentes níveis de profundidade, permitindo uma dinâmica de revisão continuada, sendo uma alternativa para alunos em recuperação ou com dificuldades (Stapleton \& Stefaniak, 2019; Bruner, 2008; Metsämuuronen \& Räsänen, 2018).

Jerome Bruner também concebeu a aprendizagem por descoberta ou instrução, que encoraja os professores a criar condições para que os alunos descubram relações entre os conceitos desenvolvidos em sala de aula, dentro da construção ativa do conhecimento, incentivando a criatividade e motivação dos alunos, por meio da solução de problemas (Stapleton \& Stefaniak, 2019; Metsämuuronen \& Räsänen, 2018; Upham et. al., 2014).

O método de resolução de problemas, também é empregado pela pedagogia libertatória de Freire, no qual educadores e educandos são críticos co-investigadores (Beckett, 2013; Freire, 2019). A teoria das competências defendida por Philippe Perrenoud retrata a capacidade de aplicação dos recursos cognitivos (saberes, informações, conhecimento prévio etc.) para solução eficiente de uma série de problemas/situações (Perrenoud, 1999; 2000). Sendo necessário respeitar os saberes do educando, considerando suas condições sociais e culturais, e aproveitar suas experiências para associar os conteúdos didáticos à realidade dos alunos (Freire, 2019).

Considerado um pesquisador interacionista, Jean Piaget acreditava que o conhecimento se desenvolve a partir da interação entre as estruturas mentais do indivíduo e a ambiente externo, sendo desenvolvida por meio de estímulos do ambiente, ou seja, o mundo, pessoas (incluindo o professor) e suas vivências (Pass, 2007). As pesquisas de Piaget foram influenciadas pelos impulsos teóricos do construtivismo e teoria dos estágios, contribuindo significativamente para a ciência do desenvolvimento cognitivo (Carey et. al., 2015).

O educador e matemático Seymour Papert, assim como Jean Piaget, defendia que os materiais propiciam ao aluno tanto o aprender-com como o aprender-sobre-o-pensar. Em outras palavras, acreditava que as ferramentas, principalmente as tecnológicas, favorecem o aprender fazendo, pois o aluno busca construir aquilo de julga significativo, envolvendo-se afetiva e 
cognitivamente com o que está sendo produzido (Castro \& Lanzi, 2017). Papert desenvolveu a teoria do Construtivismo, baseando-se na valorização do concreto, da utilização de objetos como ferramentas no processo de ensino aprendizagem durante todos os níveis escolares, objetivando romper a abordagem meramente instrucionalista de ensino (Santos, 2019).

\subsection{Robótica educacional}

O desenvolvimento tecnológico do século XXI tem modificado a forma como vivemos, pensamos e agimos. O dinamismo e o ritmo acelerado, onde novas relações sociais, familiares e de trabalho são estabelecidas a cada momento, são marcas constantes desta nova realidade. Tais mudanças também tem se manifestado nos ambientes educacionais, levando à inclusão de novos recursos pedagógicos e tecnologias a este campo. $\mathrm{O}$ uso da robótica no ambiente escolar, como recurso pedagógico e tecnológico, é resultado desta mudança.

Devido a sua natureza multidisciplinar e possibilidade de articulação entre teoria e prática, a robótica pode ser utilizada como recurso tecnológico na formação de estudantes de praticamente todos os níveis de ensino (Santos \& Sobral Júnior, 2020). A robótica tem tomado uma nova forma, deixando de ser eminentemente para a produção de robôs para se constituir um novo mediador no processo de ensino-aprendizagem (D’Abreu, 2014).

De acordo com Santos, Pozzebon e Frigo $(2013$, p. 2) "entende-se por robótica educacional a (re) utilização de conceitos de robótica industrial, em um ambiente de aprendizagem [...]", tendo como objetivo fazer uso da multidisciplinaridade destes conceitos para promover o desenvolvimento de habilidades e competências diversas que alcancem, não apenas o conhecimento técnico-científico, mas também competências individuais e sociais.

A robótica educacional é um exemplo de construção de modelo educativo inovador e eficaz em sua proposta, que permite ao aluno construir conhecimento por meio da experimentação (Fracasso et. al., 2018; Bezerra et. al., 2015). A robótica educacional como recurso pedagógico se fundamenta principalmente no Construtivismo de Piaget e no Construcionismo de Papert. Ambas as teorias sugerem que os alunos aprendem melhor quando participam ativamente no processo de aprendizagem.

O novo cenário de aprendizagem criado pela robótica educacional, possibilita que os alunos assumam o papel principal na construção do conhecimento e que o professor seja um facilitador das oportunidades de aprendizado e guia do processo (Papadakis et. al., 2016; Santoya-Mendoza et. al., 2018).

Estudos têm mostrado que as atividades de robótica educacional podem ser eficazes no desenvolvimento de habilidades como pensamento crítico, resolução de problemas, trabalho em equipe, tomada de decisão e desenvolvimento do autoconhecimento (Eguchi, 2014; Gerecke \& Wagner, 2007; Lin e.t al., 2009; Mauch, 2001). Além disso, as atividades de robótica educacional melhoram a motivação, pois oferecem às crianças a oportunidade de criar seus próprios produtos, se tornando uma maneira eficaz de aprender sobre programação e engenharia de maneira bem fundamentada e lúdica (Lin et. al., 2009; Liu et. al., 2010; Petre \& Price, 2004).

No Brasil, a robótica educacional iniciou na década de 1980 por meio da interação de profissionais brasileiros com profissionais do exterior visando o conhecimento de tipos de materiais e compreensão de suas potencialidades no ensino. Desde então, projetos de robótica educacional foram desenvolvidos pontualmente por algumas universidades brasileiras, mas foi apenas a partir dos anos 2000 que começaram a surgir espaço para a disseminação da robótica educacional em âmbito nacional, como, por exemplo, a Olimpíada Brasileira de Robótica (OBR), o fórum científico Workshop de Robótica Educacional (WRE) e a Mostra Nacional de Robótica (MNR) (Campos \& Libardoni, 2019). 


\subsection{Educação 4.0}

Em geral, toda revolução exprime um desencadear de mudanças geradas por inovações tecnológicas (Schwab, 2016). A quarta revolução industrial ou indústria 4.0 nos remete conceitos de fábricas inteligentes, propondo soluções digitais por meio de um conjunto de tecnologias capazes de associar o mundo físico, digital e biológico, impactando profundamente toda cadeia produtiva (Frank et. al., 2019). As tecnologias emergentes e disruptivas da quarta revolução industrial apresentam uma nova maneira de criar valor para as organizações e para os cidadãos, alterando significativamente a forma como vivemos, trabalhamos e nos relacionamos (Schwab, 2018).

A digitalização e a robótica marcam a quarta revolução industrial trazendo enormes efeitos sobre a educação das pessoas e novas competências profissionais (Benešová \& Tupa, 2017). A quarta revolução industrial é um vetor de transformação para o sistema educacional, numa era de inteligência artificial, robótica, big data, veículos autônomos, avanços em bio e nanotecnologia, impressão 3D, computação quântica, armazenamento de energia, ciência de materiais e avanços operacionais das redes de internet. Assim, o processo de ensino e aprendizagem demanda a quebra das barreiras impostas pela educação tradicional (Tandon \& Tandon, 2019).

A extensão da indústria 4.0 para o sistema educacional representa uma nova onda educacional no mundo, analogamente conhecida como educação 4.0. A educação 4.0 reconfigura as necessidades de aprendizagem, apresentando um novo caminho a ser trilhado pelas instituições de ensino, onde os acadêmicos não se enquadram apenas como receptores de conhecimentos, seu papel dentro das instituições de ensino será mais complexo e inovador englobando o ensino, pesquisa e aprendizagem contínua (Ishak \& Mansor, 2020).

Entre as principais discussões sobre o tema no mundo, Fisk (2019) sugere nove tendências da aprendizagem no contexto de educação 4.0, sendo elas: (1) tempo e local de aprendizagem flexível; (2) aprendizagem personalizada; (3) liberdade de escolha; (4) aprendizagem baseada em projetos; (5) experiências de campo; (6) interpretação de dados; (7) mudanças nos exames utilizados; (8) envolvimento dos alunos; e (9) Tutoria como ferramenta importante.

Apesar das perspectivas inovadoras, a educação 4.0 apresenta desafios para as instituições de ensino, que vão desde o preparo técnico dos profissionais da educação até os recursos tecnológicos para inserir seus conceitos na educação nacional. Entre os principais desafios da educação 4.0 no sistema educacional destaca-se a nova maneira de resolver problemas e necessidade de novos métodos de raciocínio para aprendizagem contínua e significativa (Ishak \& Mansor, 2020).

A Organisation for Economic Co-operation and Development (OECD) apresentou em 2016 um projeto relativo ao futuro da educação e habilidades para 2030 com o intuito de auxiliar os países no preparo de seus sistemas de ensino voltados para o futuro. As principais competências transformadoras para o futuro da educação, segundo a OECD, se concentram na utilização de ferramentas interativas, interação entre grupos heterogêneos e o agir de forma autônoma.

Os recursos tecnológicos aplicados ao ensino e aprendizagem podem ser ferramentas impulsionadoras para melhorar o processo de aprendizagem gerando um ambiente de interesse dos alunos em ser partes ativas desse processo (Halili, 2019). Os conceitos de educação 4.0 completam a integração digital na vida cotidiana, em que pessoas e máquinas se alinham para solucionar problemas, inovar e utilizar a informação onipresente e o processo de ensino e aprendizagem dinâmicos.

\section{Metodologia}

A metodologia utilizada na presente pesquisa classifica-se como bibliográfica, por realizar o levantamento de referências publicadas em diversos formatos, permitindo ao pesquisador o contato direto com o que a literatura dispõe sobre o assunto (Marconi \& Lakatos, 2018), e documental, por utilizar materiais sobre os quais podem ser aplicados tratamentos analíticos ou podem ser manipulados de acordo com o interesse de pesquisa (Gil, 2002). 
Uma análise bibliométrica foi desenvolvida visando analisar a disseminação da robótica educacional como recurso pedagógico na literatura. A bibliometria consiste em uma técnica quantitativa capaz de mensurar a produção científica. A avaliação quantitativa de materiais de cunho bibliográfico remete a ideia de tangibilidade do progresso científico, capaz de dimensionar a ciência e seu progresso (Fonseca, 1986; Bellis, 2009). Por meio da análise bibliométrica as contribuições científicas publicadas na literatura ao longo do tempo podem ser acompanhadas, tornando-se um indicador de apoio a tomada de decisão para prospecções científicas e tecnológicas.

A análise de coocorrência de palavras foi o método empregado durante a análise bibliométrica e as análises foram realizadas no software VOSviewer. De acordo com Whittaker (1989, p. 473, apud Santos, 2015, p.40) a técnica de análise de coocorrência de palavras pode ser definida como "[...] a utilização do comportamento das palavras como um meio para elucidar a estrutura das ideias e outros problemas representados em conjuntos adequados de documentos".

O objetivo da análise bibliométrica proposta foi analisar a produção científica sobre a robótica utilizada como recurso pedagógico, por meio das publicações indexadas na base de dados Web of Science (WoS). Entretanto, a produção científica das bases de dados Compendex e Scopus também foram consultadas e tiveram seus indicadores comparados aos da base de dados WoS. Os filtros utilizados durante as buscas nas bases de dados Compendex, Scopus e Web of Science foram os seguintes: termo pesquisado "Educational Robotics"; tipo de documento: todos; campo de pesquisa: título, resumo e palavras-chave; período: todos os anos; idioma: todos.

\section{Resultados e Discussão}

A pesquisa do termo "Robótica educacional" totalizou 2.019 documentos entre as bases de dados Web of Science, Scopus e Compendex, conforme ilustra a Figura 1.

Figura 1. Total de publicações por base de dados.

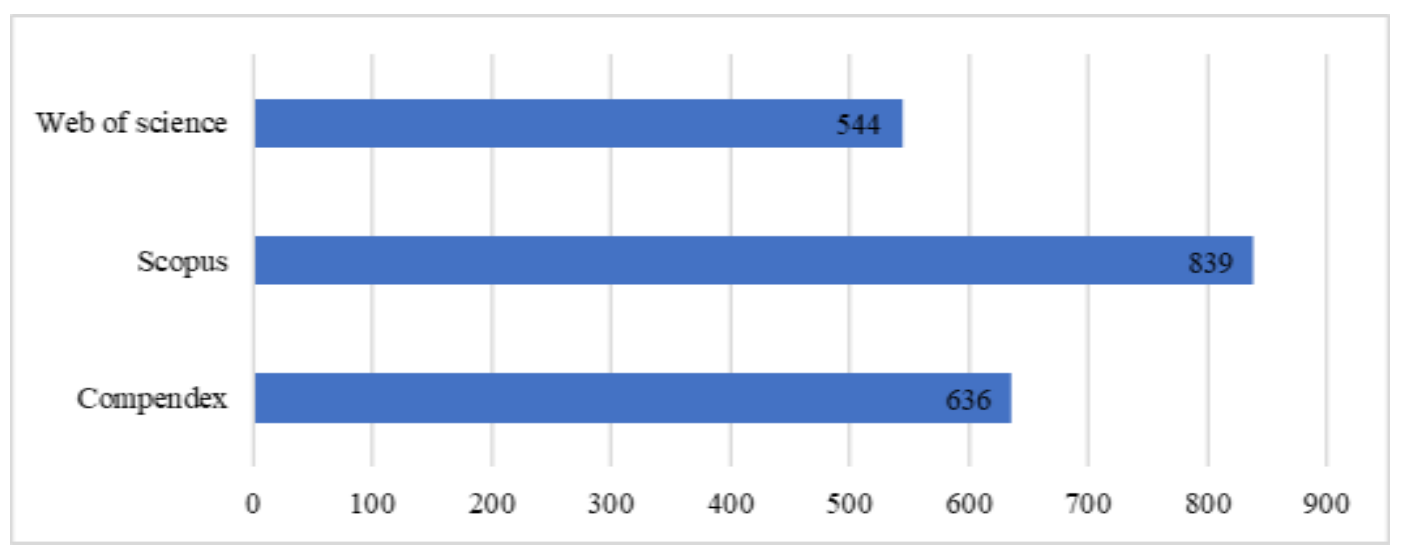

Fonte: Autores.

A base de dados Scopus possui o maior número de indexações de estudos sobre o termo robótica educacional. Na Tabela 1, têm-se os registros da base de dados classificados de acordo com os tipos de documentos publicados. 
Tabela 1. Percentual de publicações por tipo de documento.

\begin{tabular}{lccc}
\hline Publicações por tipo de documento & Compendex $(\%)$ & Scopus $(\%)$ & Web of Science $(\%)$ \\
\hline Anais de eventos & 72.6 & 59.7 & 63.4 \\
Artigos de revistas/periódicos & 21 & 29.1 & 33.1 \\
Outros & 6.4 & 11.2 & 3.5 \\
\hline
\end{tabular}

Fonte: Autores.

Observou-se que os anais de eventos representam o maior percentual de publicações entre as bases de dados, seguido de publicações de artigos em revistas e periódicos, Tabela 1.

Em 1984 têm-se o primeiro registro do termo "robótica educacional” nas bases de dados Compendex e Scopus. A publicação de Larson e Haugerud (1984), intitulada "Robótica no ensino fundamental” (Robotics in elementar education) já discutia o potencial da robótica em proporcionar uma experiência de aprendizagem enriquecedora para alunos do ensino fundamental. Até 2005 o número de publicações por ano não passava de nove e a partir de 2011 observa-se crescimento exponencial de publicações sobre o termo "robótica educacional" nas bases de dados, chegando em 2019 ao pico de publicações com 118 publicações na base de dados Compendex, 160 na Scopus e 106 na Web of Science, Figura 2.

Figura 2. Publicações por ano nas bases de dados.

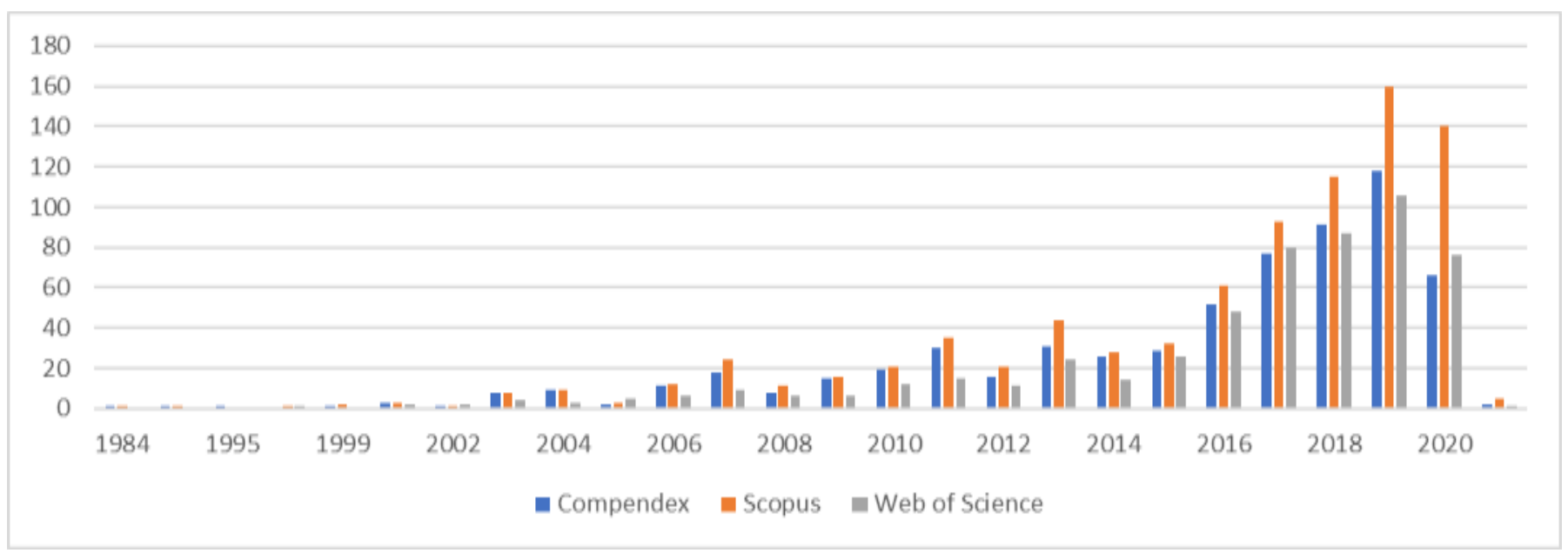

Fonte: Autores.

A década de 1980 marca o início da implantação da informática na educação, e a robótica educacional é vista como uma alternativa para diversificar a forma de utilização de computadores na educação (Silva \& Blikstein, 2020). Desde então, inúmeros avanços ocorreram favorecendo o uso de recursos tecnológicos na escola e, consequentemente, o crescimento de estudos e pesquisas sobre a robótica educacional e seus impactos na educação, sobretudo a partir dos anos 2000.

O início do século XXI ficou marcado pela preocupação de formar uma geração mais familiarizada com os recursos digitais e identificada com os desafios tecnológicos. Neste momento, a educação STEM (do inglês Science, Technologgy, Engineering and Mathematics, que pode ser traduzida como Ciência, Tecnologia, Engenharia e Matemática) começou a ganhar visibilidade.

A proposta da educação STEM é integrar os conceitos de diferentes áreas do conhecimento e a aplicação do conhecimento às situações da vida real, buscando atender à demanda por conhecimento e habilidades do século XXI.

Dentro deste cenário, a robótica, com sua natureza multidisciplinar, ganha destaque por possibilitar a criação de um ambiente adequado a compreensão de assuntos científicos e não científicos, permitir aplicações reais dos conceitos de 
engenharia e tecnologia e auxiliar na superação das dificuldades criadas pela abstração da ciência e da matemática (Peralta, 2019).

De acordo com a base de dados Web of Science as citações sobre o termo "robótica educacional" tiveram um crescimento significativo nos últimos 5 anos, passando de 68 citações por ano em 2015 para 529 citações/ano em 2020.

Figura 3. Citações do termo robótica educacional por ano - Web of Science.

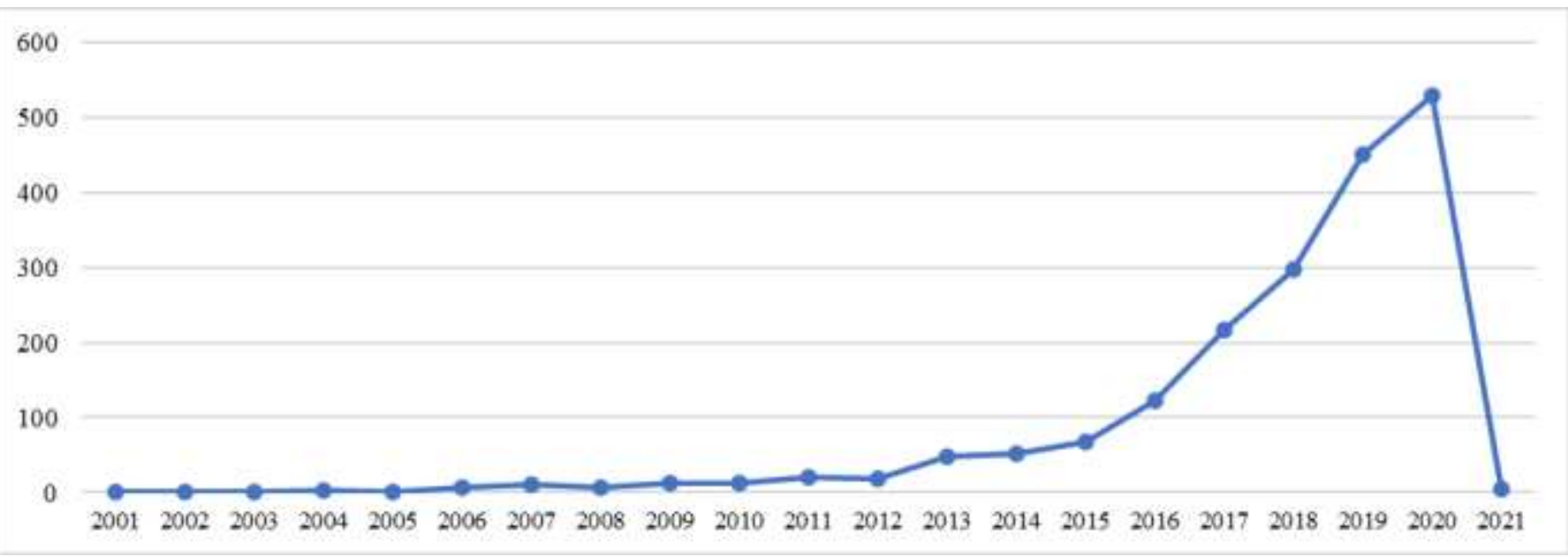

Fonte: Autores.

Outro fato que possivelmente contribuiu para o aumento no desenvolvimento de publicações científicas em robótica educacional foi o desenvolvimento de ferramentas e kits educacionais de robótica, o que tornou a robótica mais barata e acessível ao ambiente escolar. Conforme Silva e Blikstein (2020), os primeiros kits de robótica, como o Lego Mindstorms, MIT Cricket, BASIC Stamp e GoGo board, transformaram a robótica em uma realidade possível na escola.

As bases de dados Compendex, Scopus e Web of Science não possuem as mesmas subdivisões de áreas nas quais as publicações são inseridas. Entretanto, áreas de robótica, engenharia, inteligência artificial e educação são as que representam o maior percentual de publicações sobre o termo "robótica educacional" em todas as bases de dados.

A Web of Science foi selecionada para realização da análise de coocorrência de palavras por apresentar indicadores com maiores afinidades de robótica no contexto educacional. Enquanto as bases Compendex e Scopus se concentram na robótica educacional direcionada a ciência da computação, aplicações, software e engenharia, demonstrando em seus indicadores análises mais técnicas do termo. A Figura 4 apresenta as publicações por área de conhecimento da base de dados Web of Science, onde observa-se que pesquisa em robótica educacional e disciplinas científicas de educação totalizam 36\% das publicações. 
Figura 4. Publicações por área de conhecimento - Base de dados Web of Science.

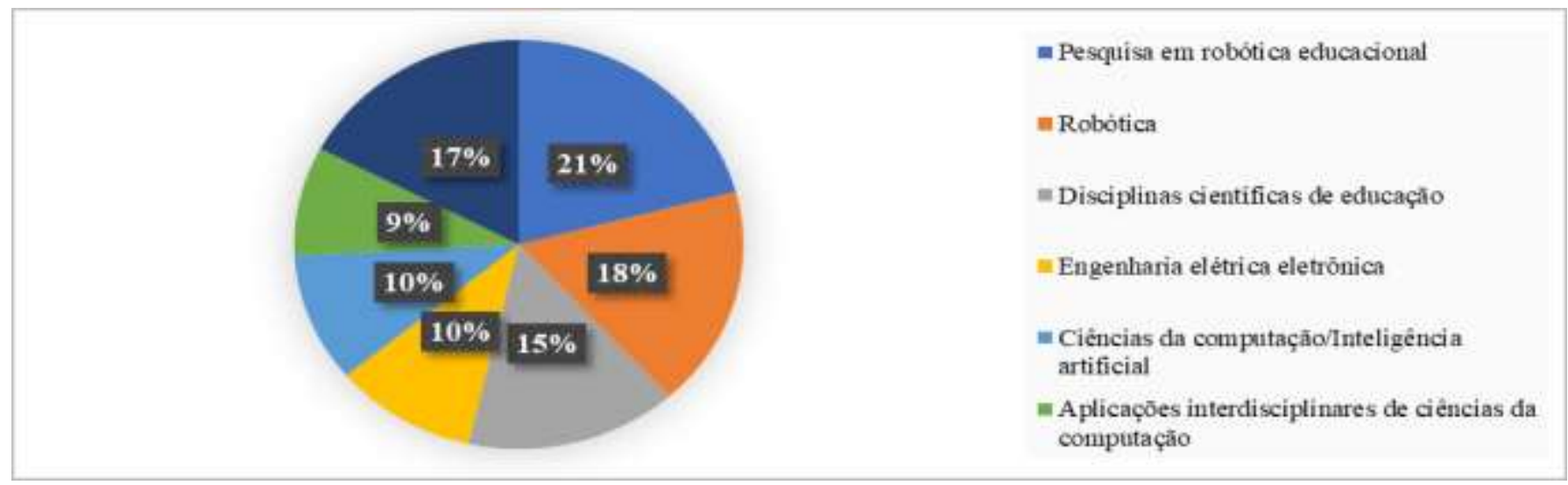

Fonte: Autores.

Com base nos indicadores das bases de dados consultadas os países com mais publicações sobre o termo "robótica educacional" são: Estados Unidos, Espanha, Itália, Brasil, Grécia e Portugal. Estando estes, entre os dez países que mais publicam no ranking das três bases de dados, Tabela 2 .

Tabela 2. Publicações por região/País.

\begin{tabular}{lclclc}
\hline \multicolumn{5}{c}{ Publicações por região/país } \\
\hline Compendex & Publicações & Scopus & Publicações & Web of Science & Publicações \\
\hline Estados Unidos & 111 & Estados Unidos & 157 & Estados Unidos & 82 \\
Brasil & 69 & Espanha & 85 & Espanha & 77 \\
Espanha & 60 & Itália & 81 & Brasil & 59 \\
Itália & 55 & Brasil & 79 & Itália & 57 \\
Grécia & 41 & Grécia & 50 & Grécia & 40 \\
Portugal & 28 & Portugal & 33 & Portugal & 25 \\
Reino Unido & 27 & Reino Unido & 33 & Áustria & 18 \\
Áustria & 23 & Rússia & 29 & Rússia & 18 \\
Rússia & 15 & Áustria & 24 & Inglaterra & 16 \\
Finlândia & 14 & Suíça & 20 & Colômbia & 15 \\
\hline
\end{tabular}

Fonte: Autores.

Entre os 2.019 documentos retornados pelas bases de dados 207 são publicações brasileiras. O Brasil tem seu primeiro registro de publicação com o termo "robótica educacional" em 2006, na base Scopus e Web of Science com o título "RoboEduc: A software for teaching robotics to technological excluded children using LEGO prototypes", dos autores Dennis Barrios-Aranibar et al. Neste trabalho os autores descrevem um software educacional para robótica desenvolvido para promover a inclusão digital. As publicações brasileiras foram alavancadas a partir de 2016, tendo seus maiores registros nas bases de dados em 2019, com 19 publicações indexadas na base de dados Compendex, 21 na Scopus e 20 na Web of Science, Figura 5. 
Figura 5. Publicações brasileiras por ano.

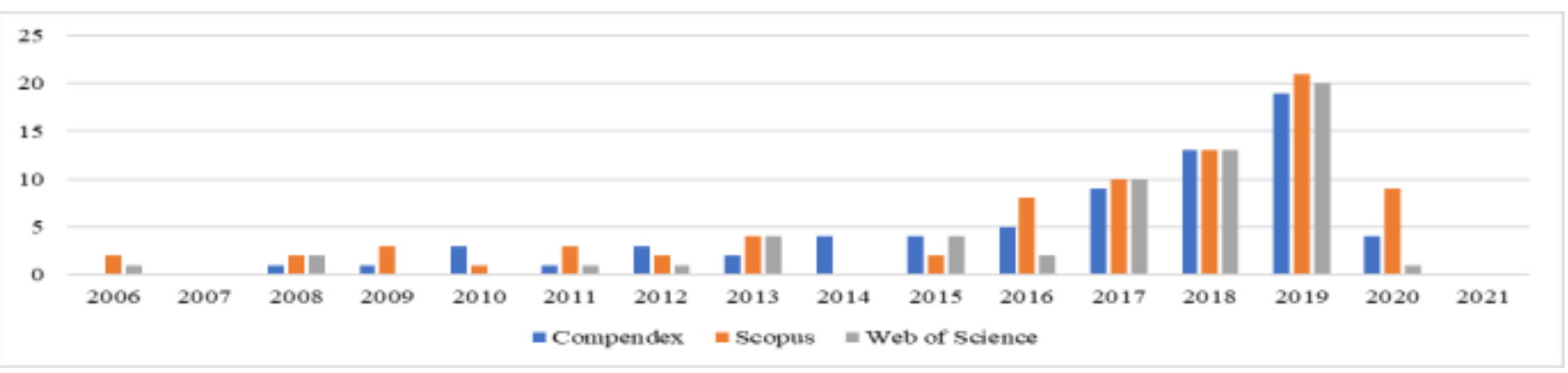

Fonte: Autores.

O inglês é considerado o idioma universal das publicações científicas, para o termo pesquisado mais de $90 \%$ das publicações foram no idioma inglês, nas três bases de dados. Há registros de publicações nos idiomas português e espanhol, entretanto o idioma inglês prevalece entre as publicações.

De acordo com as bases de dados consultadas a fonte com maior número de publicações sobre o assunto é periódico "Advances in Intelligent Systems and Computing", indexado na base de dados Scopus, Compendex, Japanese Science and Technology Agency (JST), SCImago, entre outras. O periódico possui publicações sobre aplicações, teoria e métodos de design de sistemas e computação inteligente, suas publicações não se restringem a ciências da computação possuindo publicações em praticamente todas as disciplinas como: engenharia, ciências naturais, economia, negócios, saúde, meio ambiente, entre outros.

Os periódicos "Lecture Notes In Computer Science", "Acm International Conference Proceeding Series", “Proceedings - Frontiers In Education Conference”, "Frontiers in Education Conference” e "Edulearn Proceedings” seguem o ranking de periódicos com mais publicações sobre o termo "robótica educacional". A fonte brasileira que mais publicou sobre o termo é o LARS/SBR: Latin-American Robotics Symposium / Brazilian Robotics Symposium realizado anualmente.

Os pesquisadores da Universidad Nacional de Educacion a Distancia (UNED) na Espanha - German Carro, Manuel Castro, Pedro Plaza, Elio Sancristobal e Manuel Blazques - formam uma rede de pesquisa com publicações em comum, e elencam os autores com mais publicações sobre robótica educacional nas bases de dados. As autoras Amy Eguchi e Emily Hamner filiadas ao Bloomfield College/USA e Carnegie Mellon University/USA respectivamente, também estão presentes no ranking de autores sobre o tema.

Os quatro autores brasileiros que mais publicaram sobre o tema no Brasil desenvolveram suas pesquisas junto ao Departamento de Engenharia de Computação e Automação da Universidade Federal do Rio Grande do Norte (UFRN), que possui os cursos de graduação em Engenharia de Computação e Engenharia Mecatrônica e os cursos de pós-graduação em computação e em mecatrônica. O autor Luiz M. G. Gonçalves é professor efetivo do Departamento de Engenharia de Computação e Automação da UFRN e é um dos autores do primeiro registro de publicação sobre o termo "robótica educacional" no Brasil, em 2006. O autor Aquiles M. F. Burlamaqui é professor Associado da UFRN, vinculado a graduação na ECT (Escola de Ciências e Tecnologia) e na pós-graduação no IMD (Instituto Metrópole Digital). A autora Renata P. Barros, fundou a empresa RoboEduc incubada na UFRN em 2009 e emancipada em 2011 e atua no mercado de robótica educacional. Enquanto a autora Sarah T. L. Sá atua como docente no Instituto Federal de Educação, Ciência e Tecnologia do Rio Grande do Norte, e é vice coordenadora da Olimpíada Brasileira de Robótica e membro do comitê gestor da Comissão Especial de Robótica da Sociedade Brasileira de Computação.

A National Science Foundation e European Union são os patrocinadores que mais financiam pesquisas científicas sobre o termo "robótica educacional". No Brasil as instituições que elencam as que mais investem em pesquisa sobre o tema, são: Conselho Nacional de Desenvolvimento Científico e Tecnológico, Coordenação de Aperfeiçoamento de Pessoal de Nível 
Superior, Fundação de Amparo à Pesquisa do Estado de São Paulo, Fundação de Amparo à Pesquisa do estado de Minas Gerais e Fundação de Amparo à Pesquisa do estado do Amazonas.

Tendo em conta a análise de coocorrência de palavras chaves dos dados obtidos na base de dados Web of Science e recorrendo ao software VOSviewer com o uso da técnica da coocorrência dos termos, foram identificadas 1332 palavraschaves, das quais apenas 29 possuíam um número mínimo de 10 ocorrências.

A Figura 6 ilustra os principais termos presentes nos títulos e resumos das publicações. O tamanho dos círculos representa o número de ocorrências de um termo; os maiores correspondem aos termos com maior número de ocorrências. A menor distância entre os termos indica uma relação mais forte entre eles. Os termos usados com menos frequência representam possíveis áreas de questões de pesquisa emergentes.

A partir destas considerações, foi possível observar a existência de 5 clusters que caracterizam os grupos de termos maior ocorrência nas publicações. O primeiro cluster, representado na cor vermelha, constituído por nove termos (arduino, computational thinking, constructionism, constructivism, design, learning, problem solving, robots e school), que associam a robótica educacional ao pensamento computacional, as teorias de aprendizagem construtivistas e construcionistas e ao método de ensino baseado na resolução de problemas.

Figura 6. Análise de coocorrência de palavras.

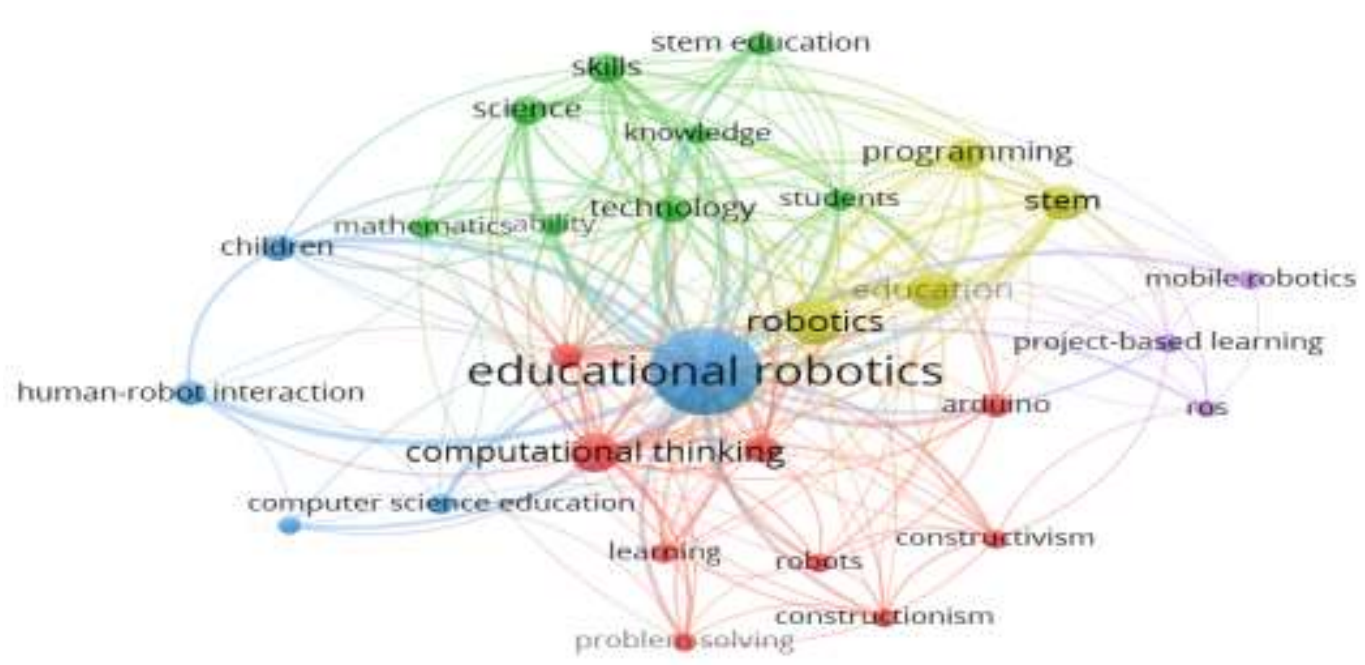

Fonte: Autores.

Observou-se que os principais anos de publicação dos termos ocorreram a partir de 2016, destacando-se os termos "educational robotics", "robotic”, “computational thinking”, "education", "steam” " "programming” como os mais citados.

Uma maneira da robótica educacional introduzir os conceitos do pensamento computacional pode ser utilizando a ferramenta Arduino. O Arduino é uma plataforma eletrônica de código e hardware aberto, baseada em software e hardware de fácil utilização, sendo ideal para construção de diversos projetos eletrônicos. Por sua vez, os outros clusters relacionam a robótica educacional ao desenvolvimento de novas habilidades por meio das metodologias STEM e STEAM. Segundo Pugliese (2020), a educação STEM se apresenta como uma proposta inovadora no ensino, dando uma ideia de rompimento com o ensino tradicional passivo, no qual a interação do aluno com o objeto de estudo é mínima e não se percebendo as conexões com o mundo empírico. Recentemente, o STEM evoluiu para STEAM, que inclui um 'A' referente às áreas da arte e design. 
Conforme Wing (2006) o pensamento computacional é uma combinação do pensamento crítico com os fundamentos, habilidades e competências comuns à área de ciências da computação, tendo a visão de que a cultura do computador ajuda a sociedade não apenas a aprender, mas oferece uma nova maneira de aprender a aprender (Lu \& Fletcher, 2009; Papert 1980).

\section{Conclusão}

A evolução tecnológica apresenta oportunidades e desafios para os diversos sistemas da sociedade, sobretudo na educação. Diante deste cenário, faz-se necessário discutir sobre os benefícios e desafios do uso das ferramentas tecnológicas na construção do conhecimento.

Com a advento da Quarta Revolução Industrial e da era digital, o ambiente escolar precisa preparar os estudantes com habilidades técnicas, cognitivas, sociais e emocionais necessárias para atender às demandas do século XXI. A educação 4.0 não apenas envolve a transformação digital do ambiente escolar, mas, sobretudo, busca repensar os processos de ensino e aprendizagem de estudantes que nasceram na era digital, considerando da incorporação de habilidades, atitudes e recursos digitais.

A utilização da robótica como recurso tecnológico no processo de ensino-aprendizagem ganhou destaque no ambiente escolar e acadêmico nos últimos anos com a disseminação de novos recursos tecnológicos, currículo e projetos voltados para esta temática. A robótica educacional é uma ferramenta capaz de introduzir a evolução tecnológica e digital no ambiente escolar por meio de uma gama de possibilidades tornando a aprendizagem dinâmica, motivadora e atrativa.

O uso da robótica com o objetivo de desenvolver o pensamento computacional possibilita a aprendizagem por meio do estímulo a capacidade de resolver problemas, em diferentes níveis de abstração e áreas do conhecimento, explorando a criatividade e a construção do saber de forma prática. Isso retoma a teoria de aprendizagem construcionista proposta por Papert, a qual implica que a construção do conhecimento deve ter como base a efetivação de uma ação concreta, que tem como resultado um produto palpável, desenvolvido com o uso de tecnologia.

Com este estudo, foi possível identificar que a robótica educacional vem ganhando relevância no contexto de educação digital e estudos relacionados. Desde a primeira publicação em 1984, a robótica educacional foi vista como um recurso pedagógico capaz de proporcionar uma experiência de aprendizagem enriquecedora.

Apesar disso, as pesquisas sobre robótica educacional só se tornaram mais numerosas a partir de 2005, acompanhando os avanços tecnológicos, as revoluções industriais e a preocupação em formar uma geração mais familiarizada com os recursos digitais. Desde 2014 observa-se um crescente número de publicações relacionadas ao tema, indicando a relevância que vem ganhando entre os pesquisadores.

Os Estados Unidos lideram entre os países com maior número de publicações sobre robótica educacional. O Brasil encontra-se entre os três primeiros países com mais publicações na área, com o total de 207 trabalhos. As publicações brasileiras começam a aparecer timidamente após 2006, mas a partir de 2016 são alavancadas. Este fato pode ser interpretado pelo surgimento de novos materiais de robótica e projetos didático-pedagógicos, bem como pela configuração das instituições de educação básica em relação ao atendimento das competências do século XXI.

A partir da análise de coocorrência pode-se identificar os principais termos referentes a robótica educacional presente na literatura. Os termos de maior ocorrência associam a robótica educacional ao pensamento computacional, as teorias de aprendizagem construtivistas e construcionistas e ao método de ensino baseado na resolução de problemas.

$\mathrm{O}$ uso da robótica educacional como recurso pedagógico capaz de proporcionar um ambiente de ensino e aprendizagem atual, eficiente e direcionado a evolução do sistema educacional apresenta tendências de crescimento. De modo geral, essa tendência reflete na inserção de novas tecnologias e recursos digitais no ambiente escolar, bem como fomenta propostas educativas multidisciplinares concretas, favorecendo a integração digital na vida cotidiana dos alunos e a 
incorporação de habilidades e atitudes capazes de prepará-los para o exercício pleno da cidadania em uma sociedade em constante evolução.

Em conclusão, a análise bibliométrica realizada neste estudo evidenciou o aumento de publicações sobre a robótica educacional, para estudos futuros sugere-se o desenvolvimento de pesquisas direcionadas a mapear quais recursos pedagógicos de robótica educacional têm sido implementados no ensino público brasileiro, em que séries/ano estão sendo inseridos e quais o impactos e/ou benefícios identificados no desenvolvimento intelectual dos alunos e na relação ensino-aprendizagem.

\section{Referências}

Beckett, K. S (2013). Paulo Freire and the Concept of Education. Educational Philosophy and Theory, 45 (1), $49-62$.

Bellis, N (2009). Bibliometrics and citation analysis. Scarecrow Press.

Benešová, A. \& Tupa, J (2017). Requirements for Education and Qualification of People in Industry 4.0. Procedia Manufacturing, 11.

Bezerra, R. P. et. al (2015). Robótica na Educação: Uma Revisão Sistemática dos Últimos 10 Anos. In: XXVI Simpósio Brasileiro de Informática na Educação.

Botomés. P. \& Kubo, O. M. (2001). Ensino-aprendizagem: uma interação entre dois processos comportamentais. Interação em psicologia, 5.

Bruner, J. S. (2008). Sobre o conhecimento: ensaios da mão esquerda. Phorte editora.

Carey, S., Zaitchik, D. \& Bascandziev, I. (2015). Theories of development: In dialog with Jean Piaget. Developmental Review.

Castro, R. M. de; \& Lanzi, L. A. C. (2017). O futuro da escola e as tecnologias: alguns aspectos à luz do diálogo entre Paulo Freire e Seymour Papert. Revista Ibero-Americana de Estudos em Educação, 1496-1510.

Coll, C. (1999). Escola e comunidade: um novo compromisso. Revista Pátio, 10.

D’ABREU, J. V. V. (2014). Robótica pedagógica: Percurso e perspectivas. Workshop de Robótica Educacional.

EGUCHI, A. (2014). Educational robotics for promoting twenty-first century skills. Journal of Automation, Mobile Robotics \& Intelligent Systems, 8 (1), 511.

Fisk, P. (2020). Education 4.0: The future of learning will be dramatically different, in school and throughout life. https://www.thegeniusworks.com/2017/01/future-education-young-everyone-taught-together/.

Fonseca, E. N. (1986). Bibliometria: Teoria e Prática. Cultrix, 1986.

Fracasso, N. et. al (2018). Análise do impacto da robótica educacional no desempenho e nas escolhas acadêmicas de alunos do ensino técnico integrado: um estudo no escopo do IFSP São Carlos. In: Workshop De Inovação, Pesquisa, Ensino E Extensão, 3, São Carlos, SP.

Frank, A. G., Dalenogare, L. S. \& Ayalam N. F. (2019). Industry 4.0 technologies: Implementation patterns in manufacturing companies. International Journal of Production Economics, 201, 15-26.

Freire, P (2019). Pedagogia da autonomia: Saberes necessários a prática educativa (60a ed). Paz \& Terra.

Führ, R. C. (2019). Educação 4.0 nos impactos da quarta revolução industrial. Appris.

Gerecke, U. \& Wagner, B. (2007). The challenges and benefits of using robots in higher education. Intell Autom Soft Comput, 13 (1), $29-43$.

Gil, A. C. (2002). Como elaborar projetos de pesquisa (4a ed.), Atlas.

Ishak, R \& Mansor, M. (2020). The Relationship between Knowledge Management and Organizational Learning with Academic Staff Readiness for Education 4.0. Eurasian Journal of Educational Research, 85, 169-184.

Halili, S. H. (2019). Technological advancements in education 4.0. Journal of Distance Education and e-Learning, 7 (1).

Lin, C. et. al (2009). A case analysis of creative spiral instruction model and students' creative problem solving performance in a Lego® robotics course. In: Chang M, Kuo R, Kinshuk, Chen G-D, Hirose M (eds) Edutainment. Springer, Heidelberg, 5670, 501-505.

Liu, E., Lin, C.\& CHANG, C. (2010). Student satisfaction and selfefficacy in a cooperative robotics course. Soc Behav Personal, 38 (8), $1135-1146$.

Lu, J. J.; Fletcher, G. H. (2009). Thinking about computational thinking. In Proc. 40th Technical Symp. on Comp. Sci. Education, 260-264. ACM.

Marconi, M. de A. \& Lakatos, E. M. (2018). Metodologia do trabalho científico (8a ed.). Atlas.

Mauch, E. (2001). Using technological innovation to improve the problem-solving skills of middle school students: educators' experiences with the LEGO Mindstorms robotic invention system. Clear House, 74 (4), 211-213. 
Research, Society and Development, v. 10, n. 4, e6310413889, 2021

(CC BY 4.0) | ISSN 2525-3409 | DOI: http://dx.doi.org/10.33448/rsd-v10i4.13889

Metsämuuronen, J. \& Räsänen, P. (2018). Cognitive-Linguistic and constructivist mnemonic triggers in teaching based on Jerome Bruner's thinking. Hypothesis and Theory, 9 .

Moreira, M. A. (1999). Teorias de aprendizagem. EPU.

Organisation for Economic Co-operation and Development (2019). Future of Education and Skills 2030. OCDE.

Papadakis, S., Kalogiannakis, M. \& Zaranis, N. (2016). Developing fundamental programming concepts and computational thinking with ScratchJr in preschool education: a case study. International Journal of Mobile Learning and Organization, 10 (3).

Papert, S. (1980). Mindstorms: children, computers, and powerful ideas. Basic Books.

Pass, S. (2007). When Constructivists Jean Piaget and Lev Vygotsky were pedagogical collaborators: A viewpoint from a study of their Communications. Journal of Constructivist Psychology, 20 (3), 277-282.

Peralta, D. A. (2019). Robótica e Processos Formativos: da epistemologia aos kits. Porto Alegre: Fi. https://www.editorafi.org/729robotica

Perrenoud, P. (2000). 10 novas competências para ensinar. Artmed.

Perrenoud, P. (1999). Profissionalização do professor e desenvolvimento de ciclos de aprendizagem. Cadernos de pesquisa,. (10), 7-26.

Petre M.\& Price, B. (2004). Using robotics to motivate 'back door’ learning. Educ Inf Technol, 9 (2), 147-158.

Pugliese, G. (2020). STEM Education - um panorama e sua relação com a educação brasileira. Currículo sem Fronteiras, 20 (12).

Santos, F. \& Sobral Júnior, G. A. (2020). Dimensão da robótica educacional como espaço educativo. Dialogia, 50-65.

Santos, R. A. dos. (2015). Análise de coocorrência de palavras na pesquisa brasileira em hiv/aids indexadas na web os science no período de 1993 -2013. (Dissertação de Mestrado) - Curso de Comunicação e Informação, Programa de Pós-Graduação em Comunicação e Informação, Universidade Federal do Rio Grande do Sul, Porto Alegre. https://lume.ufrgs.br/bitstream/handle/10183/122180/000971529.pdf?sequence=1\&isAllowed=y.

Santos, R. C. (2019). Robótica Educacional Inclusiva: uma experiência com alunos da rede pública de ensino. (Dissertação de Mestrado) - Curso de Mestrado Acadêmico em Ensino, Programa de Pós Graduação em Ensino, Universidade Estadual do Sudoeste da Bahia, Vitória da Conquista.

Santos, T., Pozzebon, E. \& Frigo, L. (2013). A utilização de robótica nas disciplinas da educação básica. In: Simpósio de Integração Científica e Tecnológica do Sul Catarinense. Anais... Araranguá: Tecnologia e Educação no Desenvolvimento Regional, 616-623.

Santoya-Mendoza, A. et. al. (2018). Robótica educativa desde la investigación como estrategia pedagógica apoyada en tic en la escuela. Cultura. Educación y Sociedad ,9(3),.699-708.

Saviani, Dermeval. (2000). Educação - Do Senso Comum à Consciência Filosófica. Coleção educação contemporânea (13 ed.). Ed. Autores Associados: Campinas-SP.

SCHWAB, K. (2016). A quarta revolução industrial. Edipro.

SCHWAB, K. \& DAVIS, N. (2018). Aplicando a quarta revolução industrial. Edipro.

Silva, R. B.; Blikstein, P. Robótica Educacional: experiências inovadoras na educação brasileira. Penso.

Stapleton, L.\& Stefaniak, J. (2019). Cognitive constructivism: Revisiting Jerome Bruner's influence on instructional design practices. TechTrends, 63.

Tandon, R. \& Tandon, S. (2020). Education 4.0: A New Paradigm in Transforming the Future of Education in India. IJISET - International Journal of Innovative Science, Engineering \& Technology, 7 (2).

Upham, P., Carney, S. \& Klapper, R. (2014). Scaffolding, software and scenarios: Applying Bruner's learning theory to energy scenario development with the public. Technological Forecasting \& Social Change, 81, 131-142.

Whittaker, J. (1989). Creativity and conformity in Science: titles, Keywords and co-word analysis. Social Studies in Science, 19 (3), 473-496.

Wing, J. M. (2006). Computational thinking. Commun. ACM, 49(3), 33 - 35. 